\title{
ASYMPTOTIC FEATURES OF VISCOUS FLOW ALONG A CORNER*
}

\author{
BY \\ ALEXANDER PAL* AND STANLEY G. RUBIN** \\ Polytechnic Institute of Brooklyn
}

\begin{abstract}
The asymptotic behavior of the equations governing the viscous flow along a right-angle corner is considered. It is demonstrated that consistent asymptotic series exist for the inner corner layer region. These expansions satisfy the corner layer equations and associated boundary conditions. They exhibit algebraic decay of all the flow properties into the boundary layer away from the corner, and prescribe algebraic decay of the cross flow velocities into the outer potential flow. Of course the streamwise velocity and vorticity are constrained to decay exponentially into the potential flow. The form of this algebraic behavior is required in order to facilitate numerical solution of the corner layer equations. Of particular significance is the use of symmetry as a means of providing a boundary condition, predicting the appearance of logarithmic terms, and specifying the occurrence of arbitrary constants. These constants can only be determined from the complete corner layer solution.
\end{abstract}

1. Introduction. The viscous flow along a corner that is formed by the intersection of two perpendicular flat plates has been studied by several authors and has recently been re-examined by Rubin [1] who discusses the past efforts on this problem in some detail. In Rubin's analysis three distinct regions were discernible (Fig. 1): an irrotational potential flow where the coordinate gradients are in most generality, of equal order; boundary layers where surface normal derivatives are much larger than either of the derivatives in the plane of the surface, and the corner layer where the $x$-wise gradient alone is small. Solutions for the various sectors were obtained by the method of matched asymptotic expansions utilizing the small parameter $\left(\nu / 2 U_{0} x\right)^{1 / 2}=R^{-1 / 2}$. $\left(U_{0}\right.$ is the velocity at $x \rightarrow-\infty)$. As is usually the case, the flow near the leading edge $x=0$ cannot be obtained in this manner and only asymptotic similarity solutions were considered.

Explicit results were presented for the first-order potential flow and first- and second-order boundary layer flows; the corner layer solution was deferred. The asymptotic behavior of the boundary- and corner-layer solutions for $\min (\eta, \zeta) \rightarrow \infty$ is directly related to the nature of the potential flow at the corner point $y^{2}+z^{2}=0$. $\eta$ and $\zeta$ are the stretched corner layer coordinates; $\eta=y R^{1 / 2} / 2 x, \zeta=z R^{1 / 2} / 2 x$. In this connection it was tacitly assumed that the potential flow is regular at $y^{2}+z^{2}=0$. However, as will be shown, this assumption is unnecessary; with the inclusion of a singularity at the corner point (first appearing in the second-order potential flow) the

* Received December 10, 1969. This research was supported by the Air Force Office of Scientific Research under Contract No. AF 49(638)-1623, Project No. 9781-01 and under Grant No. AFOSR70-1843, and monitored under the technical supervision of Major Donald L. Calvert, AFOSR.

** Currently at United Aircraft Corp., Pratt-Whitney Division, East Hartford, Connecticut. 
cross-plane corner layer velocities $v$ and $w$ need not decay exponentially when $\min (\eta, \zeta) \rightarrow \infty$, and all of the flow properties, including the vorticity, decay algebraically for $\zeta \rightarrow \infty, \eta / \zeta \rightarrow 0$, or as the flow asymptotes into the boundary layer regions.

Moreover, initial numerical solutions of the corner layer equations (2.1), using relaxation methods, strongly indicated that exponential decay into the boundary layer and potential flow was inappropriate. As it was not practical to apply the asymptotic formulas $(\zeta=\infty)$ for values of $\zeta$ in excess of ten to twenty, more relaxed boundary conditions were needed for this calculation. Such conditions are derived herein where the correct algebraic decay is described. Numerical solutions are presented by Rubin and Grossman [2].

In this paper the asymptotic behavior of the corner layer equations is considered. It is shown that separate expansions are required for $\min (\eta, \zeta) \rightarrow \infty$ (corner layer to potential flow), and $\zeta \rightarrow \infty, \eta / \zeta \rightarrow 0$ (corner layer to boundary layer) respectively, but that the two series match perfectly in an overlap domain. In Sec. 2, the equations governing the corner layer region are simplified by introducing modified cross flow velocities and a modified streamwise vorticity. Asymptotic expansions in negative integer powers of $\zeta$, for $\zeta \rightarrow \infty$, are postulated. A set of recursive partial differential equations for the asymptotic variables, as functions of $\eta$ and $\log \zeta$, is obtained. It will be shown that, assuming polynomial dependence on $\log \zeta$, the asymptotic expansion can be formally and consistently continued up to any order. Of course, the number of terms to be handled increases rapidly with the order, so that the recursion developed becomes impractical beyond a few terms. One reason for this is the appearance of constants which are undeterminable from the recursion, a situation typical of asymptotic expansions in boundary layer theory. Rubin and Grossman [2] have succeeded in obtaining the first unknown constant of the expansion numerically.

In Sec. 3, the asymptotic solution for $\min (\eta, \zeta) \rightarrow \infty$ is discussed and an interesting use of symmetry as a means of specifying a boundary condition as well as predicting the appearance of logarithmic terms is described. A compact operational calculus for the easy handling of the logarithmic terms and for reduction of the partial differential equations to formal ordinary differential equations is applied. This technique may have significance in the general asymptotic theory of solutions of differential equations. (See also Pal [3].)

In Sec. 4, the recursive equations originally of sixth order in $\eta$ are reduced to two second-order equations resulting in a considerable simplification of the system. The method of solution of this reduced set is described in Sec. 5 and a general outline of the entire procedure is given in Sec. 6. In Appendix I, the impossibility of the occurrence of fractional powers in the asymptotic expansions is proven. The existence and uniqueness of the solution is discussed in Appendix II.

2. Asymptotic expansions. The equations governing the incompressible viscous flow along a right angle corner (Fig. 1) have been discussed by several authors, including Carrier [4] and Rubin [1]. Assuming that the Reynolds number $R=2 U_{0} x / \nu$ is a large parameter, the following expansions for the velocities and pressure are appropriate for the corner layer region:

$$
\begin{aligned}
& U(x, y, z)=U_{0} u(\eta, \zeta)\left[1+O\left(R^{-1 / 2}\right)\right] \\
& V(x, y, z)=U_{0} R^{-1 / 2} v(\eta, \zeta)\left[1+O\left(R^{-1 / 2}\right)\right]
\end{aligned}
$$



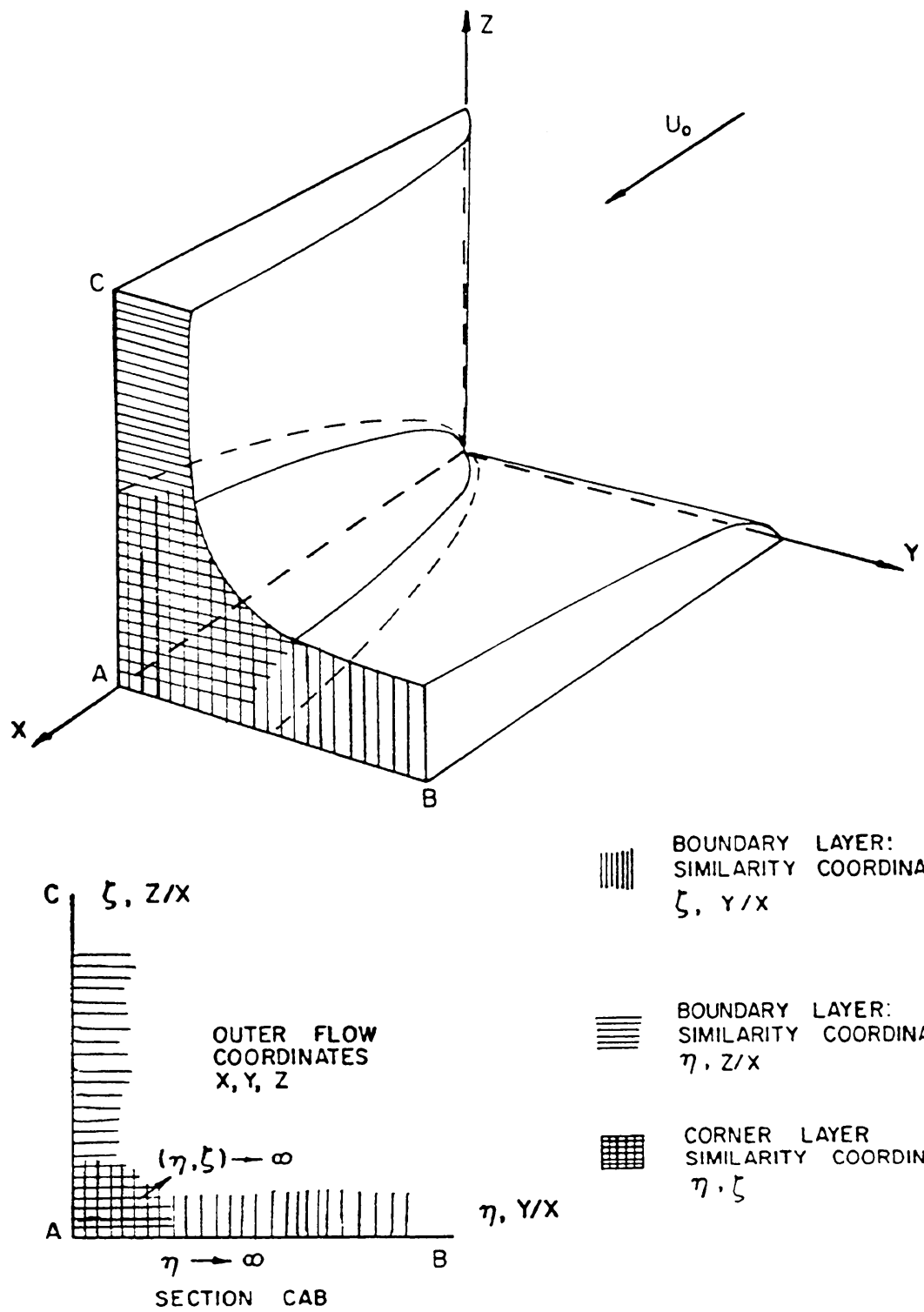

BOUNDARY LAYER:

SIMILARITY COORDINATES

S. $Y / X$

BOUNDARY LAYER:

SIMILARITY COORDINATES

$\eta, Z / X$

CORNER LAYER
SIMILARITY COORDINATES

$\eta \cdot 5$

Fig. 1. Corner flow geometry

$$
\begin{aligned}
W(x, y, z) & =U_{0} R^{-1 / 2} w(\eta, \zeta)\left[1+O\left(R^{-1 / 2}\right)\right], \\
P(x, y, z) & =p_{0}+R^{-1} p(\eta, \zeta)\left[1+O\left(R^{-1 / 2}\right)\right] .
\end{aligned}
$$

$p_{0}$ denotes the ambient uniform pressure. Similarity may be assumed for the leading nonuniform terms in the expansion on the basis of the $R \gg 1$ assumption. The stretched similarity variables are defined by $\eta=y\left(U_{0} / 2 v x\right)^{1 / 2}, \zeta=z\left(U_{0} / 2 \nu x\right)^{1 / 2}$. With the preceding expansions the Navier-Stokes equations in the region $0 \leq \eta \leq \zeta$ become, to order one, 


$$
\begin{aligned}
& -\eta u_{\eta}-\zeta u_{\zeta}+v_{\eta}+w_{\zeta}=0, \\
& -\eta u u_{\eta}-\zeta u u_{\zeta}+v u_{\eta}+w u_{\zeta}=u_{\eta \eta}+u_{\zeta \zeta}, \\
& -\eta u v_{\eta}-\zeta u w_{\zeta}+v v_{\eta}+w v_{\zeta}-u v=v_{\eta \eta}+v_{\zeta \zeta}-p_{\eta}, \\
& -\eta u w_{\eta}-\zeta u w_{\zeta}+v w_{\eta}+w w_{\zeta}-u w=w_{\eta \eta}+w_{\zeta \zeta}-p_{\zeta},
\end{aligned}
$$

(cf. Rubin, [1, Eqs. 36]). The appropriate boundary conditions for the corner geometry are $u=v=w=0$ on $\eta=0, \zeta \geq 0$, and from symmetry, $u_{\eta}=u_{\zeta}, v=w$ on $\eta=\zeta$.

In addition, for $\eta \rightarrow \infty, \eta \leq \zeta$, it should be possible to match the corner layer solution to an outer irrotational flow. The vorticity in the corner layer coordinates is

$$
\frac{U_{0}}{2 x}\left(\begin{array}{c}
w_{\eta}-v_{\zeta}+O\left(R^{-1 / 2}\right) \\
R^{1 / 2} u_{\zeta}+O\left(R^{0}\right) \\
-R^{1 / 2} u_{\eta}+O\left(R^{0}\right)
\end{array}\right)
$$

so that the leading vorticity components vanish with exponential speed if

$$
u=1+o\left(\zeta^{-N}\right), \quad w_{\eta}-v_{\zeta}=o\left(\zeta^{-N}\right)
$$

for any $N>0$, as $\zeta \rightarrow \infty$. The asymptotic solutions of Eqs. (2.1) for $\zeta \rightarrow \infty, \eta / \zeta \rightarrow 0$ have been determined by Rubin [1] and are given in the first column of Table 1.

\section{TABLE I}

First terms of the asymptotic expansions (2.8)

$n$

\begin{tabular}{l|l|l|l}
\hline & 0 & 1 & 2 \\
\hline$u_{n}$ & $f^{\prime}(\eta)$ & 0 & $\chi \eta f^{\prime \prime}(\eta)$ \\
\hline$u_{n}$ & $\eta f^{\prime}(\eta)-f(\eta)$ & 0 & $-\chi\left[-\eta^{2} f^{\prime \prime}(\eta)+3 \eta f^{\prime}(\eta)+f(\eta)\right]$ \\
\hline$x_{n}$ & & $\beta g(\eta)^{*}$ & $-4 \chi f^{\prime}(\eta)^{* *}$ \\
\hline$\varphi_{n}$ & $f(\eta)$ & 0 & $\chi\left[3 \eta f^{\prime}(\eta)+f(\eta)\right]$ \\
\hline$\psi_{n}$ & $f^{\prime}(\eta)$ & $-\beta g(\eta)$ & $\chi\left[\eta f^{\prime \prime}(\eta)+4 f^{\prime}(\eta)\right]$ \\
\hline$\theta_{n}$ & $f^{\prime \prime}(\eta)$ & $-\beta g^{\prime}(\eta)$ & $\chi f^{\prime \prime}(\eta)[-\eta f(\eta)+5]$ \\
\hline
\end{tabular}

* $g(\eta)=f^{\prime \prime}(\eta) \int_{0}^{\eta}(\tau-\beta) / f^{\prime \prime}(\tau) d \tau$. (2.4).

** $x$ is an unknown constant, obtainable from the numerical solution of the corner layer equations

The system of Eqs. (2.1) is somewhat simplified by eliminating the pressure and introducing the velocity potentials

$$
\varphi=\eta u-v, \quad \psi=\zeta u-w,
$$

and the modified vorticity

$$
\theta=\psi_{\eta}-\varphi_{\zeta}=\zeta u_{\eta}-\eta u_{\zeta}-\left(w_{\eta}-v_{\zeta}\right)
$$


These transformed variables are similar to those defined by Pearson [5] in his analysis of the corner region.

In terms of the functions $u, \theta, \varphi, \psi$, the system (2.1) becomes

$$
\begin{gathered}
\nabla^{2} u+\varphi u_{\eta}+\psi u_{\zeta}=0, \\
\nabla^{2} \theta+\varphi \theta_{\eta}+\psi \theta_{\zeta}+2 u\left(\theta-\zeta u_{\eta}+\eta u_{\zeta}\right)=0, \\
\varphi_{\eta}+\psi_{\zeta}=2 u, \\
-\varphi_{\zeta}+\psi_{\eta}=\theta
\end{gathered}
$$

and the boundary conditions transform to

$$
\begin{gathered}
u(0, \zeta)=\varphi(0, \zeta)=\psi(0, \zeta)=0, \\
u(\eta, \zeta)=1+o\left(\eta^{-N}\right) \text { if } \eta \rightarrow \infty, \quad \eta \leq \zeta, \\
\theta(\eta, \zeta)=o\left(\eta^{-N}\right) \\
\varphi(\eta, \eta)=\psi(\eta, \eta) \quad \text { from symmetry. } \\
u_{\eta}(\eta, \eta)=u_{\zeta}(\eta, \eta)
\end{gathered}
$$

The object of the following discussion is to find formal asymptotic expansions

$$
\begin{aligned}
& u \sim \sum_{0}^{\infty} u_{n} \zeta^{-\infty}, \quad \theta \sim \sum_{0}^{\infty} \theta_{n} \zeta^{-n+1} \\
& \varphi \sim \sum_{0}^{\infty} \varphi_{n} \zeta^{-n}, \quad \psi \sim \sum_{0}^{\infty} \psi_{n} \zeta^{-n+1}, \\
& v \sim \sum_{0}^{\infty} v_{n} \zeta^{-n}, \quad w \sim \sum_{1}^{\infty} w_{n} \zeta^{-n+1}
\end{aligned}
$$

valid for large values of $\zeta$, and arbitrary $\eta \leq \zeta .{ }^{1}$ The solutions for $n=0$ are related to the Blasius solution $f(\eta)$ of the two-dimensional flat plate problem (Rosenhead [7])

$$
u_{0}=f^{\prime}(\eta), \quad \theta_{0}=f^{\prime \prime}(\eta), \quad \varphi_{0}=f(\eta), \quad \psi_{0}=f^{\prime}(\eta) .
$$

The coefficients $u_{n}, \theta_{n}, \varphi_{n}, \psi_{n}$ are assumed here to depend on $\eta$ and also on $\zeta$ in a weak manner. As is frequent in asymptotic expansions, we assume that they are polynomials of $\lambda=\log \zeta$. The necessity of $(\log \zeta)^{k}$ type terms will become clear later.

Substitution of Eqs. (2.8) into (2.4) leads to the following recursive equations:

$$
\begin{aligned}
& \frac{\partial^{2} u_{n}}{\partial \eta^{2}}+f(\eta) \frac{\partial u_{n}}{\partial \eta}+f^{\prime}(\eta)\left(\frac{\partial}{\partial \lambda}-n\right) u_{n}+f^{\prime \prime}(\eta) \varphi_{n}=I_{n}, \\
& \frac{\partial^{2} \theta_{n}}{\partial \eta^{2}}+f(\eta) \frac{\partial \theta_{n}}{\partial \eta}+f^{\prime}(\eta)\left(\frac{\partial}{\partial \lambda}-n+3\right) \theta_{n}-2 f^{\prime}(\eta) \frac{\partial u_{n}}{\partial \eta}+f^{\prime \prime \prime}(\eta) \varphi_{n}+f^{\prime \prime}(\eta) \psi_{n}=J_{n}, \\
& \frac{\partial \varphi_{n}}{\partial \eta}+\left(\frac{\partial}{\partial \lambda}-n+1\right) \psi_{n}-2 u_{n}=0
\end{aligned}
$$

1 It is not evident a priori that fractional powers of 5 cannot appear in the expansions (2.8). The exclusion of noninteger powers is proven in Appendix I. For an example of the appearance of "unexpected" powers in an asymptotic expansion, see Stewartson [6] and Pal [3]. 


$$
\frac{\partial \psi_{n}}{\partial \eta}-\theta_{n}=\left(\frac{\partial}{\partial \lambda}-n+2\right) \varphi_{n-2} .
$$

Here $I_{n}, J_{v}$ depend on the functions $u_{k}, \theta_{k}, \varphi_{k}, \psi_{k}(k=0,1, \cdots, n-1)$ :

$$
\begin{aligned}
I_{n}= & -\left(\frac{\partial}{\partial \lambda}-n+1\right)\left(\frac{\partial}{\partial \lambda}-n+2\right) u_{n-2}-\sum_{k=1}^{n-1}\left\{\varphi_{n-k} \frac{\partial}{\partial \eta}+\psi_{n-k}\left(\frac{\partial}{\partial \lambda}-k\right)\right\} u_{k}, \\
J_{n}=- & \left(\frac{\partial}{\partial \lambda}-n+2\right)\left(\frac{\partial}{\partial \lambda}-n+3\right) \theta_{n-2}+2 \eta \sum_{k=1}^{n-2} u_{n-k-2}\left(\frac{\partial}{\partial \lambda}-k\right) u_{k} \\
& -\sum_{k=1}^{n-1}\left\{\varphi_{n-k} \frac{\partial}{\partial \eta}+\psi_{n-k}\left(\frac{\partial}{\partial \lambda}-k+1\right)\right\} \theta_{k}-2 \sum_{k=1}^{n-1}\left(\theta_{k}-\frac{\partial u_{k}}{\partial \eta}\right) u_{n-k} .
\end{aligned}
$$

In Eqs. (2.10) any sum with an upper limit less than one is defined to be zero. The system (2.9) is supplemented by the definitions

$$
\begin{aligned}
v_{n} & =\eta u_{n}-\varphi_{n}, \\
w_{n} & =u_{n}-\psi_{n}
\end{aligned}
$$

obtained from (2.2) and (2.3).

Eqs. (2.9) are partial differential equations, and formally they are not any simpler than Eqs. (2.4) from which they were derived. However, the dependence of all unknowns on the variable $\lambda$ is partially known. All quantities appearing in these equations are polynomials in $\lambda$, the coefficients of which are functions of $\eta$. This is certainly true of $u_{0}, \theta_{0}, \varphi_{0}, \psi_{0}$, (which do not depend on $\lambda$ ) and can be proven by induction for all $u_{n}, \theta_{n}, \varphi_{n}, \psi_{n}$. In fact, these special solutions can be derived explicitly from the solution of the reduced system from which the $\partial / \partial \lambda$ terms are omitted. The algorithm of solving such partial differential equations is introduced and discussed in detail elsewhere (cf. Pal [3]).

The system (2.9) will be solved recursively, with the inductive hypothesis that the functions $u_{k}, \theta_{k}, \varphi_{k}, \psi_{k}$ are known for $k=0, \cdots, n-1$. In fact these functions have been determined for $k=0,1,2$ explicitly, in terms of the Blasius function $f(\eta)$. These expressions, listed in Table 1, can be verified by substitution into the recursive equations and boundary conditions.

The first few terms of the asymptotic series do not depend on $\lambda$, since the governing equations and boundary conditions can be satisfied with the omission of the $\partial / \partial \lambda$ terms. The governing equations for $\varphi_{1}$ and $u_{1}$ uncouple so that with $I_{1}=J_{1}=0$

$$
\begin{gathered}
\varphi_{1}^{\prime \prime \prime}+f(\eta) \varphi_{1}^{\prime \prime}-f^{\prime}(\eta) \varphi_{1}^{\prime}+2 f^{\prime \prime}(\eta) \varphi_{1}=0, \\
2 u_{1}=\varphi_{1}^{\prime}, \\
\varphi_{1}(0)=\varphi_{1}^{\prime}(0)=0, \quad \varphi_{1}^{\prime}=o\left(\eta^{-N}\right) \text { as } \eta \rightarrow \infty, \text { for any } N>0 .
\end{gathered}
$$

The primes denote differentiations with respect to $\eta$. The only solution of this system of equations and boundary condition is $\varphi_{1}=u_{1} \equiv 0$ (cf. Libby and Fox [8]). Therefore $I_{2}=J_{2}=0$ and the first nonvanishing $I_{k}, J_{k}$ become

$$
\begin{aligned}
& I_{3}=2 \psi_{1} u_{2}=-2 \chi \eta \beta g f^{\prime \prime} \\
& J_{3}=-\varphi_{2} \theta_{1 \eta}+\psi_{1} \theta_{2}-2 \theta_{1} u_{2}=\beta \chi\left[g^{\prime \prime}\left(3 \eta f^{\prime}+f\right)+2 g^{\prime} \eta f^{\prime \prime}-g\left(5 f^{\prime \prime}-\eta f f^{\prime \prime}\right)\right],
\end{aligned}
$$

where $g=g(\eta)=f^{\prime \prime}(\eta) \int_{\eta}^{\infty}\left([\tau-\beta] / f^{\prime \prime}(\tau)\right) d \tau$ and $\chi$ is an undetermined constant. 
3. Symmetry as a boundary condition. With the boundary conditions (2.6a) and (2.6b) and from the expansions (2.8) we must have as $\eta \rightarrow \infty$

$$
\begin{array}{ll}
u_{n}(\eta, \lambda)=o\left(\eta^{-N}\right) & (n=1,2,3, \cdots), \\
\theta_{n}(\eta, \lambda)=o\left(\eta^{-N}\right) & (n=0,1,2,3, \cdots),
\end{array}
$$

for any $N>0$. Consequently from (2.9c) and (2.9d) it follows that

$$
\begin{gathered}
\partial \varphi_{n} / \partial \eta+(\Delta-n+1) \psi_{n}=o\left(\eta^{-N}\right), \\
\partial \psi_{n} / \partial \eta=(\Delta-n+2) \varphi_{n-2}+o\left(\eta^{-N}\right), \quad(\Delta \equiv \partial / \partial \lambda),
\end{gathered}
$$

or by (3.1a), (2.11a) and (2.11b)

$$
\begin{gathered}
\partial v_{n} / \partial \eta+(\Delta-n+1) w_{n}=o\left(\eta^{-N}\right) \quad(n=1,2, \cdots) \\
-\partial w_{n} / \partial \eta+(\Delta-n+2) v_{n-2}=o\left(\eta^{-N}\right), \quad(n=1,2 \cdots) .
\end{gathered}
$$

From the asymptotic formulas for the Blasius function and the expressions of Table 1 we find that

$$
\begin{aligned}
v_{0}(\eta) & =\beta+o\left(\eta^{-N}\right), & v_{1}(\eta) & =o\left(\eta^{-N}\right), \\
w_{1}(\eta) & =\beta+o\left(\eta^{-N}\right), & w_{2}(\eta) & =-4 \chi+o\left(\eta^{-N}\right) .
\end{aligned}
$$

For $n>1$, Eqs. (3.3) can be solved recursively. Thus it can be shown by induction that

$$
\begin{aligned}
& v_{n}(\eta, \lambda)=P_{n}(\eta, \lambda)+o\left(\eta^{-N}\right), \\
& w_{n}(\eta, \lambda)=Q_{n}(\eta, \lambda)+o\left(\eta^{-N}\right),
\end{aligned}
$$

where $P_{n}(\eta, \lambda), Q_{n}(\eta, \lambda)$ are polynomials of $\eta$ which satisfy the recursive equations

$$
\begin{aligned}
& \partial P_{n} / \partial \eta=-(\Delta-n+1) Q_{n}, \\
& \partial Q_{n} / \partial \eta=(\Delta-n+2) P_{n-2} .
\end{aligned}
$$

It will be assumed that $P_{n}, Q_{n}$ are polynomials of $\lambda=\log \zeta$ as well, of orders to be determined later. We will call $P_{n}(\eta, \lambda), Q_{n}(\eta, \lambda)$ the "polynomial part" of $v_{n}(\eta, \lambda), w_{n}(\eta, \lambda)$.

The polynomial parts of the functions $u_{n}, \varphi_{n}, \psi_{n}, \theta_{n}$ can be defined similarly. By Eqs. (3.1) the polynomial parts of $u_{n}, \theta_{n}$ are identically 0 , with the exception of $u_{0}$ for which the polynomial part is unity. Further, because of $(2.11), P_{n}(\eta, \lambda)$ is the polynomial part of $-\varphi_{n}(\eta, \lambda)$ and $Q_{n}(\eta, \lambda)$ the polynomial part of $-\psi_{n}(\eta, \lambda)$. The polynomial parts of the variables $u_{n}, v_{n}, w_{n}, \varphi_{n}, \psi_{n}, \theta_{n}$ for $n=0,1,2$, are listed in Table 2.

With the use of the polynomial parts, the asymptotic expansions (2.8) of the functions $v, w$, can be written for large $\zeta$ and large $\eta$ in the form

$$
\begin{aligned}
v & \sim \sum_{0}^{\infty} P_{n}(\eta, \lambda) \zeta^{-n} \\
w & \sim \sum_{1}^{\infty} Q_{n}(\eta, \lambda) \zeta^{-n+1}
\end{aligned}
$$

More precisely $v=\sum_{0}^{m} P_{n}(\eta, \lambda) \zeta^{-n}+o\left(\zeta^{-m}\right)+o\left(\eta^{-N}\right)$ and similarly for $w$.

For $\eta \rightarrow \infty, \eta \leq \zeta$, the functions $v$ and $w$ satisfy, by Eqs. (2.4) and (2.6),

$$
\partial v / \partial \eta+\partial w / \partial \zeta=o\left(\eta^{-N}\right), \quad-\partial v / \partial \zeta+\partial w / \partial \eta=o\left(\eta^{-N}\right)
$$


TABLE 2

Polynomial parts of $u_{n}, \cdots \theta_{n}$.

\begin{tabular}{|c|c|c|c|}
\hline \multicolumn{4}{|c|}{$n$} \\
\hline & 0 & 1 & 2 \\
\hline$u_{n}$ & 1 & 0 & 0 \\
\hline$v_{n}$ & $\beta$ & 0 & $-\chi(4 \eta-\beta)$ \\
\hline$w_{n}$ & & $\beta$ & $-4 x$ \\
\hline$\varphi_{n}$ & $\eta-\beta$ & 0 & $\chi(4 \eta-\beta)$ \\
\hline$\psi_{n}$ & 1 & $-\beta$ & $4 x$ \\
\hline$\theta_{n}$ & 0 & 0 & 0 \\
\hline
\end{tabular}

TABLE 3

Boundary conditions oblained from the symmetry condition.

\begin{tabular}{c|c|c}
\hline$n$ & $Q_{n}(0, \lambda)=$ \\
\hline 1 & $=P_{0}(0, \lambda)=-\beta$ & \\
\hline 2 & $=4 \chi$ & $\chi$ arbitrary constant \\
\hline 3 & $=-P_{2}(0, \lambda)=-\beta \chi$ & \\
\hline 4 & $=0$ & \\
\hline 5 & $=P_{4}(0, \lambda)$ & $\begin{array}{c}P_{5}(0, \lambda)=\text { constant } \\
\chi^{\prime} \text { arbitrary constant }\end{array}$ \\
\hline 6 & $=P_{5}(0, \lambda) \lambda+\chi^{\prime}$ & \\
\hline
\end{tabular}

Therefore, it may be assumed that the expansions (3.8) of $v, w$ can be rearranged into a series of pairwise conjugate harmonic functions. If the complex independent variable $\tau=e^{-i x / 4}(\eta+i \zeta)$ is introduced, then the rearranged expansion for $v-i w$ will consist of analytic terms. To be compatible with (3.8), we must set for $\eta \rightarrow \infty$, $\zeta \geq \eta$

$$
e^{i \approx / 4}[v(\eta, \zeta)-i w(\eta, \zeta)] \sim(2)^{1 / 2} \beta+\sum_{n=1}^{\infty} \frac{\gamma_{n}(\log \tau)}{\tau^{n}},
$$

where the $\gamma_{n}(\Lambda)$ are polynomials. By applying the symmetry condition

$$
v(\eta, \eta)=w(\eta, \eta)
$$


to the asymptotic expansion (3.9), we find that (3.10) is equivalent to the condition that in (3.9) all polynomials $\gamma_{n}(\Lambda)$ have only real coefficients.

In order to obtain relations between the polynomial parts of $v_{n}$, wn we have to reintroduce the variables $\eta, \zeta$ into (3.9) and examine the behavior of (3.9) for $\eta / \zeta \rightarrow 0$. Therefore, we substitute $\log \tau=\lambda+\omega(\eta / \zeta)$, where

$$
\omega(t)=i \pi / 4+\log (1-i t) .
$$

From here it follows by Taylor's theorem that

$$
\gamma_{n}(\log \tau)=\sum_{k=0}^{\infty} \frac{1}{k !}\left(\frac{\partial}{\partial \lambda}\right)^{k} \gamma_{n}(\lambda) \omega(\eta / \zeta)
$$

With the notation $\partial / \partial \lambda=\Delta$, this can be written formally by the use of $(3.11)$ in the form

$$
\gamma_{n}(\log \tau)=\exp [\omega(\eta / \zeta) \Delta] \gamma_{n}(\lambda)=\exp [i(\pi / 4) \Delta]\left(1-i \frac{\eta}{\zeta}\right) \Delta \gamma_{n}(\lambda)
$$

The last expression and

$$
\tau^{-n}=\exp [-i n \pi / 4] \zeta^{-n}(1-i \eta / \zeta)^{-n}
$$

are substituted into (3.9). This yields

$$
v-i w \sim(1-i) \beta+\sum_{n=1}^{\infty}\left\{\exp [i(\pi / 4)(\Delta-n)]\left(1-i \frac{\eta}{\zeta}\right)^{\Delta-n} \gamma_{n}(\lambda)\right\} \zeta^{-n}
$$

From here by the application of the binomial series we find

$$
v-i w \sim(1-i) \beta+\sum_{n=1}^{\infty}\left[P_{n}^{*}(\eta, \lambda)-i Q_{n+1}^{*}(\eta, \lambda)\right] \zeta^{-n}
$$

where

$$
P_{n}^{*}-i Q_{n+1}^{*}=\sum_{r=0}^{n} \exp \left\{i \frac{\pi}{4}(\Delta-n-r-1)\right\}\left(\begin{array}{c}
\Delta-n \\
r
\end{array}\right) \gamma_{n-r}(\lambda) \eta^{r}
$$

(here $\Delta$ is assumed real). Series expansion of a function $F(\Delta)$ in powers of $\Delta$ is a legitimate operation, as the operator $F(\Delta)=F(\partial / \partial \lambda)$ is always applied to polynomials of $\lambda$, and therefore, the series expansion breaks off after a finite number of terms. (See also Pal [3].)

The $n$th order approximations $V_{n}, W_{n}$ of the series (3.8), satisfy the asymptotic relations

$\partial V_{n} / \partial \eta-\partial W_{n} / \partial \zeta=o\left(\eta^{-N}\right)+O\left(\zeta^{-n-1}\right), \quad \partial V_{n} / \partial \zeta+\partial W_{n} / \partial \eta=o\left(\eta^{-N}\right)+O\left(\zeta^{-n-1}\right)$.

Therefore, the right-hand sum of (3.13) formally satisfies the Cauchy-Riemann equations and $P_{n}^{*}, Q_{n}^{*}$ satisfy Eqs. (3.6). From this it follows that

$$
P_{n}^{*}(\eta, \lambda)=P_{n}(\eta, \lambda), \quad Q_{n}^{*}(\eta, \lambda)=Q_{n}(\eta, \lambda)
$$

if and only if

$$
P_{n}(0, \lambda)-i Q_{n+1}(0, \lambda)=P_{n}^{*}(0, \lambda)-i Q_{n+1}^{*}(0, \lambda)
$$

or by (3.14)

$$
P_{n}(0, \lambda)-i Q_{n+1}(0, \lambda)=\exp [((\partial / \partial \lambda)-n-1) i(\pi / 4)] \gamma_{n}(\lambda),
$$


$(n=1,2, \cdots)$. From here,

$$
\gamma_{n}(\lambda)=\exp [-((\partial / \partial \lambda)-n-1) i(\pi / 4)]\left[P_{n}(0, \lambda)-i Q_{n+1}(0, \lambda)\right] .
$$

The coefficients $\gamma_{n}(\lambda)$ must be real by the symmetry as discussed previously; hence

$$
\begin{aligned}
& \operatorname{Im}\left\{\exp \left[i \frac{\pi}{4}\left(n+1-\frac{\partial}{\partial \lambda}\right)\right]\left[P_{n}(0, \lambda)-i Q_{n+1}(0, \lambda)\right]\right\} \\
& =\sin \left[\frac{\pi}{4}\left(n+1-\frac{\partial}{\partial \lambda}\right)\right] P_{n}(0, \lambda)-\cos \left[\frac{\pi}{4}\left(n+1-\frac{\partial}{\partial \lambda}\right)\right] Q_{n+1}(0, \lambda)=0,
\end{aligned}
$$

and with the notations $P_{n}(0, \lambda)=p_{n}=p_{n}(\lambda), Q_{n}(0, \lambda)=q_{n}=q_{n}(\lambda)$,

$$
q_{n}(\lambda)=\tan \left[\frac{\pi}{4}\left(n-\frac{\partial}{\partial \lambda}\right)\right] p_{n-1}(\lambda) \equiv J_{n} p_{n-1}(\lambda) .
$$

This is the boundary condition obtainable from the condition of symmetry in terms of the polynomial parts of $Q_{n}, P_{n-1}$ of $\psi_{n}$ and $\varphi_{n-1}$. The operator $J_{n}$ can be obtained explicitly by the Taylor series expansion of the function $\tan (n(\pi / 4)-t)$ as follows:

$$
\begin{aligned}
& J_{0}=-\frac{\pi}{4} \Delta-\frac{1}{3}\left(\frac{\pi}{4} \Delta\right)^{3}-\frac{2}{15}\left(\frac{\pi}{4} \Delta\right)^{5}-\cdots \\
& J_{1}=1-2\left(\frac{\pi}{4} \Delta\right)+2\left(\frac{\pi}{4} \Delta\right)^{2}-\frac{8}{3}\left(\frac{\pi}{4} \Delta\right)^{3}+\cdots \\
& J_{2}=\left(\frac{\pi}{4} \Delta\right)^{-1}-\frac{1}{3}\left(\frac{\pi}{4} \Delta\right)-\frac{1}{45}\left(\frac{\pi}{4} \Delta\right)^{3}-\cdots \\
& J_{3}=-1-2\left(\frac{\pi}{4} \Delta\right)-2\left(\frac{\pi}{4} \Delta\right)^{2}-\frac{8}{3}\left(\frac{\pi}{4} \Delta\right)^{3}-\cdots
\end{aligned}
$$

and $J_{4 m+}=J_{\nu}$ if $m, \nu$ are integers.

Clearly $J_{\nu}$ reduces to an at most $k$ th order differential operator if applied to a $k$ th order polynomial in $\lambda$. The first term in the expansion of $J_{2}$ requires integration of the operand, with an arbitrary integration constant. Hence $Q_{4 m+2}(0, \lambda)$ is determined by the symmetry condition only up to an arbitrary constant. On the other hand, the order of $Q_{4 m+2}(0, \lambda)$ exceeds the order of $P_{4 m+1}(0, \lambda)$ by one.

The appearance of logarithmic terms can be thus considered a necessary consequence of the symmetry condition. Since $\varphi_{1}(\eta, \lambda)$ vanishes identically, the first logarithmic term can be expected to appear in $Q_{6}(\lambda)$.

4. Reduction of the perturbation system (2.9). Substitution of (2.9c) and (2.9d) into (2.9a) and (2.9b) leads to

$$
\begin{array}{r}
\varphi^{\prime \prime \prime}+f \varphi^{\prime \prime}+f^{\prime} \delta \varphi^{\prime}+2 f^{\prime \prime} \varphi+(\delta+1)\left(\psi^{\prime \prime}+f \psi^{\prime}+\delta f^{\prime} \psi\right)=2 I_{n}, \\
-f^{\prime} \varphi^{\prime \prime}+f^{\prime \prime \prime} \varphi+\psi^{\prime \prime \prime}+f \psi^{\prime \prime}+2 f^{\prime} \psi^{\prime}+f^{\prime \prime} \psi \\
\quad=J_{n}+(\delta+2)\left[\varphi_{n-2}^{\prime \prime}+f \varphi_{n-2}^{\prime}+f^{\prime}(\delta+3) \varphi_{n-2}\right], \\
f \equiv f(\eta), \quad \varphi \equiv \varphi_{n}(\eta, \lambda), \quad \psi \equiv \psi_{n}(\eta, \lambda), \quad \delta \equiv \delta_{n}=\Delta-n .
\end{array}
$$

We introduce the new functions

$$
\Phi(\eta, \lambda)=\varphi_{n}(\eta, \lambda)-P_{n}(\eta, \lambda), \quad \Psi(\eta, \lambda)=\psi_{n}(\eta, \lambda)-Q_{n}(\eta, \lambda),
$$


so that Eqs. (4.1a), (4.1b) become

$$
\begin{gathered}
\Phi^{\prime \prime \prime}+f \Phi^{\prime \prime}+\delta f^{\prime} \Phi^{\prime}+2 f^{\prime \prime} \Phi+(\delta+1)\left[\Psi^{\prime \prime}+f \Psi^{\prime}+\delta f^{\prime} \Psi\right]=e_{n}, \\
-f^{\prime} \Phi^{\prime \prime}+f^{\prime \prime \prime} \Phi+\Psi^{\prime \prime \prime}+f \Psi^{\prime \prime}+2 f^{\prime} \Psi^{\prime}+f^{\prime \prime} \Psi=g_{n},
\end{gathered}
$$

where

$$
\begin{gathered}
e_{n}=2 I_{n}-2 f^{\prime \prime} P_{n}, \\
g_{n}=J_{n}-f^{\prime \prime \prime} P_{n}-f^{\prime \prime} Q_{n}+(\delta+2)\left[\Phi_{n-2}^{\prime \prime}+f \Phi_{n-2}^{\prime}+(\delta+3) f^{\prime} \Phi_{n-2}\right], \\
{\left[P_{k}=P_{k}(\eta, \lambda), \quad Q_{k}=Q_{k}(\eta, \lambda)\right] .}
\end{gathered}
$$

The expressions (4.4) are obtained by taking (3.6) into consideration. The transformation (4.2) has the advantage that, as can be shown by induction, the functions $e_{n}, g_{n}$ and the unknowns $\Phi, \psi$ decay exponentially, or more precisely

$$
\begin{aligned}
\Phi & =O\left(\eta^{M} \exp \left[-(\eta-\beta)^{2} / 2\right)\right], & \Psi & =O\left(\eta^{M} \exp \left[-(\eta-\beta)^{2} / 2\right)\right], \\
e_{n} & =O\left(\eta^{M} \exp \left[-(\eta-\beta)^{2} / 2\right)\right], & g_{n} & =O\left(\eta^{M} \exp \left[-(\eta-\beta)^{2} / 2\right)\right],
\end{aligned}
$$

for $\eta \rightarrow \infty$, where $M$ is a positive number dependent on $n$ alone; $\beta$ is the familiar constant related to the Blasius function $f(\eta)$; i.e., $\beta=\lim _{\eta \rightarrow \infty}\left(\eta f^{\prime}(\eta)-f(\eta)\right)$.

The polynomials $P_{n}(\eta, \lambda), Q_{n}(\eta, \lambda)$ are determined by the recursive relations (3.6a), (3.6b) up to their constant terms, since $P_{0}(\eta, \lambda), \cdots, P_{n-1}(\eta, \lambda) ; Q_{1}(\eta, \lambda), \cdots, Q_{n-1}(\eta, \lambda)$ are the polynomial parts of $\varphi_{0}, \cdots, \varphi_{n-1}, \psi_{1}, \cdots, \psi_{n-1}$ respectively and therefore known by the inductive assumption.

The constant term $q_{n}(\lambda)$ of $Q_{n}(\eta, \lambda)$ is prescribed by the symmetry condition (3.16). Thus the $\lambda$-polynomial $P_{n}(0, \lambda)=p_{n}(\lambda)$ is the only unknown part of (4.4a) and (4.4b), since by (3.6a)

$$
P_{n}(\eta, \lambda)-P_{n}(0, \lambda)=-\int_{0}^{\eta}(\Delta-n+1) Q_{n}(\tau, \lambda) d \tau \equiv P_{n}^{* *}(\eta, \lambda)
$$

is known. Thus we may write (4.4a), (4.4b) in the form

$$
e_{n}=E_{n}-2 f^{\prime \prime}(\eta) p_{n}(\lambda), \quad g_{n}=G_{n}-f^{\prime \prime \prime}(\eta) p_{n}(\lambda),
$$

where $E_{\mathrm{n}}, G_{\mathrm{n}}$ are known quantities:

$E_{n}=2 I_{n}-2 f^{\prime \prime}(\eta) P_{n}^{* *}(\eta, \lambda)$,

$G_{n}=J_{n}-f^{\prime \prime \prime}(\eta) P_{n}^{* *}(\eta, \lambda)-f^{\prime \prime}(\eta) Q_{n}(\eta, \lambda),+(\delta+2)\left[\Phi_{n-2}^{\prime \prime}+f \Phi_{n-2}^{\prime}+(\delta+3) f^{\prime} \Phi_{n-2}\right]$.

Equation (4.3b) can be once integrated:

$$
-f^{\prime} \Phi^{\prime}+f^{\prime \prime} \Phi+\Psi^{\prime \prime}+f \Psi^{\prime}+f^{\prime} \Psi=l_{n}+C
$$

where

$$
l_{n}=L_{n}-f^{\prime \prime}(\eta) p_{n}(\lambda), \quad L_{n}=-\int_{\eta}^{\infty} G_{n}(\tau, \lambda) d \tau .
$$

The integration constant $C$ must vanish, as $L_{n}$ and the left-hand side of (4.6) are both exponentially small as $\eta \rightarrow \infty$.

We now introduce the new variable

$$
s=\Phi^{\prime}+(\delta-1) \Psi .
$$


The combination of Eqs. (4.3a), (4.6) (the latter with $C=0$ ) and (4.8) leads to

$$
\Phi^{\prime \prime \prime}+f \Phi^{\prime \prime}+\delta f^{\prime} \Phi^{\prime}-(\delta-1) f^{\prime \prime} \Phi=s^{\prime \prime}+f s^{\prime}+f^{\prime} s-(\delta-1) l_{n}
$$

and

$$
(\delta-1)\left\{s^{\prime \prime}+f s^{\prime}+(\delta+2) f^{\prime} s-e_{n}+2 l_{n}\right\}=0 .
$$

Multiplication of (4.10) by $(\delta-1)^{-1}$ and consideration of (4.5), (4.7) results in

$$
s^{\prime \prime}+f s^{\prime}+(\delta+2) f^{\prime} s=E_{n}-2 L_{n} .
$$

(Multiplication by $(\delta-1)^{-1}$ is for $n \neq 1$ a uniquely defined operation, if applied to polynomials of $\lambda .(\delta-1)^{-1}$ satisfies the identity

$$
\left.(\delta-1)^{-1}(\delta-1)=(\delta-1)(\delta-1)^{-1}=1 .\right)
$$

Eq. (4.9) can be reduced to second order with the relation

$$
\Phi=f^{\prime}(\eta) \Omega(\eta, \lambda)-p_{n}(\lambda)
$$

and the new variable

$$
t_{n}(\eta, \lambda)=t(\eta, \lambda)=\Omega^{\prime}(\eta, \lambda) .
$$

Thus we find that (4.9) transforms to

$$
f^{\prime} t^{\prime \prime}+\left(f f^{\prime}+3 f^{\prime \prime}\right) t^{\prime}+\left(\delta f^{\prime 2}-f f^{\prime \prime}\right) t=E_{n}-(\delta+1) L_{n}-(\delta+1) f^{\prime} s .
$$

We note that by definition $\Phi$ is obtainable from $t$ by

$$
\Phi(\eta, \lambda)=f^{\prime}(\eta) \int_{0}^{\eta} t(\tau, \lambda) d \tau-p_{n}(\lambda)+k(\lambda) f^{\prime}(\eta) .
$$

$k_{n}(\lambda)$ is an unknown polynomial of $\lambda$ to be determined from the boundary conditions. $\Psi(\eta, \lambda)$ is obtained from $\Phi$ and $s$ by (4.8).

5. Boundary conditions and asymptotic behavior. The unknowns $\varphi_{n}, \psi_{n}$ satisfy at the wall $\eta=0$ the boundary conditions

$$
\begin{gathered}
\varphi_{n}(0, \lambda)=0, \\
\varphi_{n}^{\prime}(0, \lambda)=0, \\
\psi_{n}(0, \lambda)=0,
\end{gathered}
$$

which are equivalent to $u_{n}(0, \lambda)=v_{n}(0, \lambda)=w_{n}(0, \lambda)=0$.

In terms of the unknowns $\Phi, \Psi$, we find by virtue of the symmetry condition that

$$
\begin{aligned}
\Phi(0, \lambda) & =-P_{n}(0, \lambda)=-p_{n}(\lambda), \\
\Psi(0, \lambda) & =-Q_{n}(0, \lambda)=-J_{n} p_{n-1}(\lambda) \equiv-q_{n}(\lambda), \\
\Phi^{\prime}(0, \lambda) & =-P_{n}^{\prime}(0, \lambda)=-(\Delta-n+1) q_{n}(\lambda) \equiv-r_{n}(\lambda) .
\end{aligned}
$$

For the unknowns $s, t$, considering (4.8) and (4.12), these equations become

$$
\begin{gathered}
s(0, \lambda)=-2(\Delta-n) q_{n}(\lambda), \\
k_{n}(\lambda)=-(1 / \kappa) r_{n}(\lambda), \quad\left(\kappa=f^{\prime \prime}(0)\right)
\end{gathered}
$$


and

$$
\lim _{\eta \rightarrow 0}[\eta t(\eta, \lambda)]=0 .
$$

It may be observed that on the basis of the analysis of the singularity of (4.13) at $\eta=0$, (5.5) may be replaced by the weaker

$$
\eta^{2} t \rightarrow 0 \text {. }
$$

Now we turn our attention to the asymptotic behavior of $s$ and $t$ as $\eta \rightarrow \infty$. By the Prandtl-Goldstein principle it must be possible to match the corner layer equations, up to exponentially small terms, to an irrotational and symmetrical outer flow. From irrotationality it is necessary and sufficient that $u_{n}(\eta, \lambda)$ and $\theta_{n}(\eta, \lambda)$ tend to 0 with exponential speed. Equivalently, the outer flow is irrotational if and only if

$$
\begin{aligned}
& \Phi(\eta, \lambda)=o\left(\eta^{-N}\right), \\
& \Psi(\eta, \lambda)=o\left(\eta^{-N}\right) .
\end{aligned}
$$

In fact, if the latter conditions are satisfied, then the former are also satisfied by Eqs. (3.6), (2.4c, d); and vice versa. The symmetry condition was expressed in terms of the polynomials $P_{n}, Q_{n}$ in Eqs. (3.16). It is desirable to express these boundary conditions for $\eta \rightarrow \infty$ solely in terms of the functions $s_{n}, t_{n}$. By Eq. (4.8)

$$
s(\eta, \lambda)=o\left(\eta^{-N}\right), \quad(\eta \rightarrow \infty) .
$$

By (4.12) we must have

$$
\Omega(\eta, \lambda)=p_{n}(\lambda)+o\left(\eta^{-N}\right)
$$

hence

$$
t(\eta, \lambda)=o\left(\eta^{-N}\right) \quad(\eta \rightarrow \infty)
$$

is a plausible assumption. From (4.8), (4.14) and (5.4) it follows that

$$
\begin{aligned}
& \Phi(\eta, \lambda)=f^{\prime}(\eta)\left\{\int_{0}^{\eta} t(\tau, \lambda) d \tau-(1 / k) p_{n}(\lambda)\right\}-p_{n}(\lambda), \\
& \Psi(\eta, \lambda)=(\delta-1)^{-1}\left\{f^{\prime \prime}(\eta)\left[-\int_{0}^{\eta} t(\tau, \lambda) d \tau+(1 / \kappa) r_{n}(\lambda)\right]+s(\eta, \lambda)-f^{\prime}(\eta) t(\eta, \lambda)\right\},
\end{aligned}
$$

where $r_{n}(\lambda)$ was defined in (5.2c).

The boundary condition (5.6b) is satisfied if (5.7), (5.8) are satisfied. (5.6a) requires that

$$
p_{n}(\lambda)=\int_{0}^{\infty} t(\eta, \lambda) d \eta-\frac{1}{\kappa} r_{n}(\lambda)
$$

(5.12) completes the set of equations required to determine the new unknowns $s, t, p_{n}$, $q_{n}, r_{n}$. Indeed, $q_{n}$ is known from (3.16), $r_{n}$ from (5.2c), hence $p_{n}$ can be determined from (5.11), once $t \equiv t_{n}$ is known. It remains to see that the differential equation (4.11) with the corresponding boundary conditions (5.3), (5.6a) (Problem A) and (once $s \equiv s_{n}$ is known) the differential equation (4.13) with its boundary conditions (5.5),(5.6b) (Problem B) have unique solutions. This is shown in Appendix II.

It is remarkable that neither of the problems A, B contain the polynomials $p_{n}, q_{n}, r_{n}$ 
in their formulations. This fortunate circumstance allows the separation of the determination of $p_{n}$ from the solution of Problems A and B.

6. Summary of the recursive construction of the asymptotic expansion. In the preceding sections a complete set of equations and unknowns were derived for the recursive construction of the asymptotic series of the flow velocities. For the reader's convenience we list here the relevant unknowns and equations in the order they are required in the construction (as opposed to the order of their derivation).

List of unknowns. Unknowns corresponding to a particular order $m>1$ : (All quantities are polynomials of $\lambda=\log \zeta$. Dependence on $\lambda$ will not be noted, in general, explicitly. The operator $\partial / \partial \eta$ will be replaced by the symbol $D$ and $\partial / \partial \lambda$ by $\Delta$.)

(a) $u_{m}, v_{m}, w_{m}, \varphi_{m}, \psi_{m}, \theta_{m}$ functions of $\eta$.

(b) $\Phi_{m}, \Psi_{m}$ : the "asymptotic" parts of $\varphi_{m}, \psi_{m}$.

(A function $h(\eta)$ defined in $(0, \infty)$ will be termed "asymptotic" if a $k>0$ exists such that $|h(\eta)|=O\left(\eta^{k} \exp \left[-(\eta-\beta)^{2} / 2\right]\right)$ as $\eta \rightarrow \infty$.) $-P_{m}=\varphi_{m}-\Phi_{m},-Q_{m}=\psi_{m}-\Psi_{m}$ are the polynomial parts of $\varphi_{m}$ and $\psi_{m}$ respectively.

(c) The constants $P_{m}, q_{m}, r_{m}$ are defined by the equations $p_{m}=P_{m}(0), q_{m}=Q_{m}(0)$, $r_{m}=P_{m}^{\prime}(0)$,

(d) $s_{m}(\eta)=\Phi_{m}^{\prime}+(\Delta-m-1) \Psi_{m}, t_{m}(\eta)=D\left(\Phi_{m} / f^{\prime}\right)$.

(e) Auxiliary functions; $A_{m}, B_{m}, E_{m}, G_{m}, I_{m}, J_{m}, L_{m}$.

These quantities are known for $m=1,2$. If they are assumed known for $m=$ $1, \cdots, n-1$, then they can be obtained for $n-m$ by the following steps:

List of equations and sequence of solutions.

1. Evaluate the auxiliary functions $I_{n}, J_{n}$, which depend only on $u_{m}, v_{m}, v_{m}, \varphi_{m}$, $\psi_{m}, \theta_{m}$ for $m=0,1, \cdots, n-1$ by the use of Eqs. $(2.10) ; I_{m}, J_{m}$ are asymptotic.

2. Evaluate the constants

$$
q_{n}=-\cot [[\pi / 4](n-\Delta)] P_{n-1}, \quad r_{n}=(\Delta-n+1) q_{n},
$$

and the polynomials

$$
\begin{aligned}
Q_{n}(\eta) & =(\Delta-n+2) \int_{0}^{\eta} P_{n-2}(\tau) d \tau+q_{n}, \\
P_{n}^{* *}(\eta) & =-(\Delta-n+1) \int_{0}^{\eta} Q_{n}(\tau) d \tau .
\end{aligned}
$$

3. Calculate the asymptotic functions

$$
\begin{aligned}
& E_{n}(\eta)=2 I_{n}-2 f^{\prime \prime} P_{n}^{* *}, \\
& G_{n}(\eta)=J_{n}-f^{\prime \prime \prime} P_{n}^{* *}-f^{\prime \prime} Q_{n}+(\Delta-n-2)\left[\Phi_{n-2}^{\prime \prime}+f \Phi_{n-2}^{\prime}+(\Delta-n+3) f^{\prime} \Phi_{n-2}\right] \\
& L_{n}(\eta)=-\int_{\eta}^{\infty} G_{n}(\tau) d \tau \\
& A_{n}(\eta)=2 I_{n}-2 f^{\prime \prime} P_{n}^{* *}-2 L_{n}
\end{aligned}
$$

4. Problem A. Solve the equation

$$
s_{n}^{\prime \prime}+f s_{n}^{\prime}+(\Delta-n+2) f^{\prime} s_{n}=A_{n}
$$

subject to the boundary conditions:

$$
s_{n}(0)=-2 r_{n}, \quad s_{n} \text { asymptotic. }
$$

Obtain $B_{n}=E_{n}-(\Delta-n+1)\left(L_{n}-f^{\prime} s_{n}\right)\left(B_{n}(\eta)\right.$ is asymptotic $)$. 
5. Problem B. Solve the equation

$$
f^{\prime} t_{n}^{\prime \prime}+\left(f f^{\prime}+3 f^{\prime \prime}\right) t_{n}^{\prime}+\left[(\Delta-n) f^{\prime}-f f^{\prime \prime}\right] t_{n}=B_{n}
$$

subject to the boundary conditions

$$
\lim _{n \rightarrow 0} \eta t_{n}(\eta)=0, \quad t_{n} \quad \text { asymptotic. }
$$

6. Evaluate the constant

$$
p_{n}=\int_{0}^{\infty} t_{n}(\eta) d \eta-(1 / \kappa) r_{n} \quad\left(\kappa=f^{\prime \prime}(0)\right)
$$

and set $P_{n}(\eta)=P_{n}^{* *}(\eta)+p_{n}$.

7. Evaluate

$$
\begin{aligned}
\Phi(\eta) & =-f^{\prime}(\eta) \int_{\eta}^{\infty} t_{n}(\zeta) d \zeta-p_{n}\left[1-f^{\prime}(\eta)\right] \\
\Psi(\eta) & =(\Delta-n-1)^{-1}\left[s_{n}(\eta)-\Phi_{n}^{\prime}(\eta)\right] \\
\varphi_{n}(\eta) & =\Phi(\eta)+P_{n}(\eta) \\
\psi_{n}(\eta) & =\Psi(\eta)+Q_{n}(\eta) \\
u_{n}(\eta) & =\frac{1}{2}\left[\varphi_{n}^{\prime}(\eta)+(\Delta-n+1) \psi_{n}(\eta)\right] \\
\theta_{n}(\eta) & =\psi_{n}^{\prime}-(\Delta-n+2) \varphi_{n-2}(\eta) \\
v_{n}(\eta) & =\varphi_{n}(\eta)-\eta u_{n}(\eta) \\
w_{n}(\eta) & =u_{n}(\eta)-\psi_{n}(\eta)
\end{aligned}
$$

7. Summary. It is demonstrated that apart from a sequence of arbitrary constants a unique asymptotic series of the type (2.14) exists which satisfies the corner layer equations (2.1) and the appropriate boundary conditions of the corner geometry. This series is continuable to arbitrary order by use of the operator calculus that has been introduced herein. The algebraic decay of all the corner flow properties into the boundary layers $(\zeta \rightarrow \infty, \eta / \zeta \rightarrow 0)$ is established, as is the algebraic decay of the crosswise velocities into the potential flow $(\eta \rightarrow \infty, \eta \leq \zeta)$. This asymptotic behavior is important in the numerical solution of the corner layer equations presented by Rubin and Grossman [2]. The use of symmetry as a boundary condition and the appearance of logarithmic terms is formally demonstrated.

Appendix I. Exclusion of the possibility of fractional powers in the asymptotic expansions. We assume that the unknowns $u, v, w, \varphi, \psi, \theta$ have asymptotic expansions of the form

$$
\begin{aligned}
& u \sim f^{\prime}(\eta)+\sum_{1}^{\infty} \tilde{u}_{n} \zeta^{-\mu_{\infty}}, \\
& v \sim \eta f^{\prime}(\eta)-f(\eta)+\sum_{1}^{\infty} \tilde{v}_{n} \zeta^{-\mu_{n}}, \\
& w \sim \sum_{1}^{\infty} \tilde{w}_{n} \zeta^{1-\mu_{n}},
\end{aligned}
$$




$$
\begin{aligned}
\varphi & \sim f^{\prime}(\eta)+\sum_{0}^{\infty} \varphi_{n} \zeta^{-\mu_{n}}, \\
\psi & \sim \zeta f^{\prime}(\eta)+\sum_{0}^{\infty} \psi_{n} \zeta^{1-\mu_{n}}, \\
\theta & \sim \zeta f^{\prime \prime}(\eta)+\sum_{1}^{\infty} \tilde{\theta}_{n} \zeta^{1-\mu_{n}},
\end{aligned}
$$

where $\left\{\mu_{k}\right\}$ is an increasing sequence such that $\mu_{k} \rightarrow \infty$ as $k \rightarrow \infty$. Suppose that there are noninteger values $\mu_{k}$, and $\mu_{r}=\mu$ is the smallest among them. $\mu$ must be greater than or equal to one, since $0<\mu=\mu_{1}<1$ would imply by (2.2) that the leading term of the expansion of $w$ is of order $\zeta^{1-\mu}$, where $1-\mu>0 . u_{n}, \theta_{n}, \varphi_{n}, \psi_{n}$ satisfy the Eqs. (2.9) with the following modifications:

(1) substitute $n=r$,

(2) $I_{n}, J_{n}, \varphi_{n-2}$ are replaced by zero;

(3) the term $\partial / \partial \lambda-n$ is everywhere replaced by $\partial / \partial \lambda-\mu$.

Since the expansion of $\varphi$ does not contain a $\zeta^{1-\mu}$ term and that of $\psi$ a $\zeta^{2-\mu}$ term, we find from the modified equation (2.9d) $Q_{r}(\eta, \lambda)=-q(\lambda)$ and from the modified Eq. (2.9c)

$$
P_{r}(\eta, \lambda)=-(\partial / \partial \lambda-\mu+1) q(\lambda)-p(\lambda) .
$$

By the modified Eq. (3.16) then

$$
Q_{r}(0, \lambda)=p(\lambda)=0 .
$$

This condition obviously assures that the $O\left(|\boldsymbol{\tau}|^{-\mu+1}\right)$ term of the external field is symmetric.

The variables $\tilde{\Phi}=\varphi_{n}-P_{n}, \tilde{\Psi}=\psi_{n}-Q_{n}$ can be introduced as before and then they satisfy the modified homogeneous equations (4.3a) and (4.6) (in which $e_{n}=0$, $l_{n}+C=0$ and $\delta$ in (4.3a) has the meaning $\partial / \partial \lambda-\mu$ ). If the variables $\xi, z$ are introduced by equations analogous to (4.8) and (4.12) (with $p_{n}(\lambda)=0$ ), and (4.12a) then $\xi, \tau$ satisfy the modified, homogeneous system (4.11) and (4.13), i.e.,

$$
\begin{gathered}
\tilde{s}^{\prime \prime}+f \tilde{s}^{\prime}+(\partial / \partial \lambda-\mu+2) f^{\prime} s=0, \\
f^{\prime} \tilde{t}^{\prime \prime}+\left(f f^{\prime}+3 f^{\prime \prime}\right) \tilde{t}^{\prime}+\left[(\partial / \partial \lambda-\mu) f^{\prime 2}-f f^{\prime \prime}\right] \tilde{t}=\tilde{s}^{\prime \prime}+f \xi^{\prime}+f^{\prime} \xi
\end{gathered}
$$

The corresponding boundary conditions are also homogeneous:

$$
\begin{aligned}
& \tilde{s}_{r}(0, \lambda)=0 \text {, } \\
& \tilde{s}_{r}(\eta, \lambda)=o\left(\eta^{-N}\right) \quad(\eta \rightarrow \infty), \\
& z_{r}(\eta, \lambda)=O\left(\eta^{-1}\right) \quad(\eta \rightarrow 0), \\
& t_{r}(\eta, \lambda)=o\left(\eta^{-N}\right) \quad(\eta \rightarrow \infty) .
\end{aligned}
$$

$\tilde{s}$ and $\tau$ are assumed to be polynomials of $\lambda$. Let the highest order terms of these polynomials be denoted by $\hat{s}(\eta), \hat{t}(\eta)$. Then $\hat{s}, \hat{t}$ satisfy (I.1) and (I.2) in which the $\partial / \partial \lambda$ terms are omitted.

Suppose first that $\hat{s}(\eta)$ does not vanish identically. Then $\hat{s}(\eta)$ satisfies the eigenvalue problem (I.1), (I.3) where $\partial / \partial \lambda$ is replaced by 0 in (I.1). Since $2-\mu$ is the lowest eigenvalue by assumption, the corresponding eigenfunction, $\hat{s}(\eta) \neq 0$ for $0<\eta<\infty$. (See Courant and Hilbert [10].) We may assume $\hat{s}(\eta)>0$ without restriction of generality.

From (I.1) we derive by integration

$$
(\mu-1) \int_{0}^{\infty} f^{\prime}(\eta) \hat{s}(\eta) d \eta=\left[\hat{s}^{\prime}(\eta)+f(\eta) \hat{s}(\eta)\right]_{0}^{\infty}=-\hat{s}^{\prime}(0) .
$$


The left-hand integral is positive, since $\hat{s}(\eta)>0$ for $\eta>0$. For the same reason $\hat{s}^{\prime}(0)>0$. Therefore $\mu<1$ follows. But $\mu<1$ is unacceptable, so that $\hat{s}(\eta)$ must vanish identically. Thus, all solutions of the eigenvalue problem (I.1)-(I.4) must be of the form $\hat{s}=0$ and therefore also $\tilde{s}=0$. The leading term $\hat{t}(\eta)$ of $\tilde{t}(\eta, \lambda)$ must then satisfy by (I.2)

$$
f^{\prime} \hat{t}^{\prime \prime}+\left(f f^{\prime}+3 f^{\prime \prime}\right) \hat{t}^{\prime}-\left(\mu f^{\prime 2}+f f^{\prime \prime}\right) \hat{t}=0
$$

with the homogeneous boundary conditions (I.4). However, Libby and Fox [8] have shown that this eigenvalue problem does not have any positive eigenvalues $\mu$. Thus also $\hat{t} \equiv 0$ and thus $\tilde{t} \equiv 0$. Substitution of $z \equiv 0, \hat{t} \equiv 0$ leads to $u_{n} \equiv 0, \theta_{n} \equiv 0, \varphi_{n} \equiv 0$, $\psi_{n} \equiv 0$, a contradiction to the assumption of the existence of a noninteger eigenvalue.

Appendix II. Existence and uniqueness of the solutions of Problems A and B.

Problem A. We first note that both Eq. (4.11) and the boundary conditions (5.3) are inhomogeneous. By a substitution $s=S+\alpha f^{\prime \prime}+\beta f^{\prime \prime \prime}$ with properly chosen constants $\alpha, \beta$ Problem A can be brought into the form

$$
\begin{gathered}
S^{\prime \prime}+f S^{\prime}-(n-\Delta-2) f^{\prime} S=a(\eta) \\
S(0)=0, \quad S \text { asymptotic. }
\end{gathered}
$$

Furthermore, the inhomogeneous term $a(\eta)$ may satisfy the boundary conditions

$$
a(0)=0, \quad a(\eta) \text { asymptotic. }
$$

Then the transformation $s=\left(f^{\prime \prime} / f^{\prime}\right)^{1 / 2} \sigma$ will take the equation into the formally selfadjoint form

$$
\mathbf{L} \sigma-(n-\Delta-2) \sigma=a\left(f^{\prime} f^{\prime \prime}\right)^{-1 / 2} \equiv A(\eta)
$$

where

$$
\mathrm{L}=\left(f^{\prime \prime} / f^{\prime}\right)^{1 / 2} \mathrm{D}\left[\left(1 / f^{\prime \prime}\right) \mathrm{D}\left[\left(1 / f^{\prime \prime}\right) \mathrm{D}\left(f^{\prime \prime} / f^{\prime}\right)^{1 / 2}\right]\right] .
$$

The operator $L$ is defined over the dense subspace $D^{\prime}$ of $\mathcal{L}_{2}(0, \infty)$ characterized as follows: $u$ belongs to $D^{\prime}$ if and only if $u$ is twice differentiable in $[0, \infty)$ and

(a) $u \in \mathscr{L}_{2}(0, \infty)$,

(b) $\left(f^{\prime \prime}\right)^{-1 / 2} \mathrm{D}\left[\left(f^{\prime \prime} / f^{\prime}\right)^{1 / 2} u\right] \in \mathfrak{L}_{2}(0, \infty)$,

(c) $\mathbf{L} u \in \mathfrak{L}_{2}(0, \infty)$,

(d) $\lim _{\eta \rightarrow 0}\left(\eta^{-1 / 2} u\right)=0$.

Then it can be shown by application of the theory of closed operators in a Hilbert space (cf. Dunford and Schwartz, [10, Vol. 2, Chaps. XII, XIII]) that L can be extended into a strictly self-adjoint operator over a space $\mathbb{D}$ such that $D^{\prime} C D c \mathfrak{L}_{2}(0, \infty)$.

The operator $L$ is negative, i.e. for any $u$ in $D$

$$
\begin{aligned}
(u, \mathrm{~L} u) & =\int_{0}^{\infty}\left(f^{\prime \prime} / f^{\prime}\right)^{1 / 2} u \mathrm{D}\left[\left(1 / f^{\prime \prime}\right) \mathrm{D}\left(f^{\prime \prime} / f^{\prime}\right) u\right] d \eta \\
& =-\int_{0}^{\infty}\left(1 / f^{\prime \prime}\right)\left\{\mathrm{D}\left[\left(f^{\prime \prime} / f^{\prime}\right) u\right]\right\}^{2} d \eta \leq 0 .
\end{aligned}
$$

Therefore, the spectrum of $\mathbf{L}$ is a subset of the closed negative half-axis. Consequently the resultant $R_{\mu}$ of the operator $\mathbf{L}$ (i.e. the inverse of $\mathbf{L}-\mu \mathrm{I}$ ) is a bounded operator for any $\mu>0 . \mathbf{R}_{\mu}$ can be expressed with the Green's function $G_{\mu}(\eta, \zeta)$ of the differential operator $\mathbf{L}-\mu \mathrm{I}$ subject to the conditions (a) and (d): $\mathbf{R}_{\mu} u=\int_{0}^{\infty} G_{\mu}(\eta, \zeta) u(\zeta) d \zeta$. The operator $\mathbf{R}_{\mu}$ can be obtained by series expansion if $\mathbf{R}_{\mu}$ is known, for values of $\mu$ which 
do not belong to the spectrum of $L$. In fact, by the binomial series

$$
\mathbf{R}_{\mu-\Delta}=(\mathrm{L}-\mu \mathrm{I}-\Delta \mathrm{I})^{-1}=\sum_{0}^{\infty}(\mathrm{L}-\mu \mathrm{I})^{-k-1} \Delta^{k}=\sum_{0}^{\infty} \mathbf{R}_{\mu}^{k+1} \Delta^{k} .
$$

The right-hand series is always finite for $\Delta=\partial / \partial \lambda$, if the left-hand operator is applied to polynomials of $\lambda$; therefore the equation is rigorously valid.

The solution of Problem A can be stated thus as

$$
\sigma=\sum_{k=0}^{\infty} \mathrm{R}_{n-2}^{k+1}(\partial / \partial \lambda)^{k} A(\eta, \lambda), \quad s=\left(f^{\prime \prime} / f^{\prime}\right)^{1 / 2} \sigma+\alpha f^{\prime \prime}+\beta f^{\prime \prime \prime} .
$$

This solution is by virtue of its derivation unique.

Problem B. The method of solution is based on a construction similar to the one applied to Problem A. First a transformation $t=T+\alpha f^{\prime \prime}+\beta f^{\prime \prime \prime}$ is applied so that the new inhomogeneous term is $O\left(\eta^{2}\right)$ for $\eta \rightarrow 0$. Then a transformation $T=\left(f^{\prime \prime}\right)^{1 / 2}\left(f^{\prime}\right)^{-2} \tau$ brings the differential equation (4.13) into the formal self-adjoint form $\mathbf{M}_{\tau}$ $(n-\Delta) \tau=B$ where

$$
\mathbf{M} z \equiv\left[\left(f^{\prime \prime}\right)^{1 / 2} /\left(f^{\prime}\right)^{2}\right] \mathbf{D}\left\{\left[\left(f^{\prime 3}\right) / f^{\prime \prime}\right] \mathbf{D}\left[-\left[\left(f^{\prime \prime}\right)^{1 / 2} /\left(f^{\prime}\right)^{2}\right] z\right]-\left[f f^{\prime \prime} /\left(f^{\prime}\right)^{2}\right] z\right\},
$$

and $B$ is square integrable in $(0, \infty) . M$ is defined over the space $D^{\prime \prime} c \mathcal{L}_{2}(0, \infty)$ of all functions $z(\eta)$ which have in $[0, \infty]$ piecewise continuous second derivatives and are such that

(a) $z \in \mathfrak{L}_{2}(0, \infty)$,

(b) $\left(\left(f^{\prime}\right)^{3 / 2} /\left(f^{\prime \prime}\right)^{1 / 2}\right) \mathrm{D}\left[\left(\left(f^{\prime \prime}\right)^{1 / 2} /\left(f^{\prime}\right)^{2}\right) z\right] \in \mathfrak{L}_{2}(0, \infty)$,

(c) $M u \in \mathfrak{L}_{2}(0, \infty)$.

(A boundary condition at $\eta=0$ is not necessary, because (b) implies by Schwarz's inequality that $\eta^{-1 / 2} z(\eta) \rightarrow 0$ as $\eta \rightarrow 0$. Thus the function $z(\eta)$ defined by (II.4) satisfies $z(\eta)=O\left(\eta^{-3 / 2}\right)$, a boundary condition stronger than $\left(5.5^{*}\right)$.)

Again $\mathbf{M}$ can be extended into a self-adjoint operator over a dense subspace $\tilde{D}$ of $\mathcal{L}_{2}(0, \infty)$ and the spectrum of $M$ can be shown to consist of nonpositive real values. Therefore the solution of the equation $[\mathbf{M}-(n-\Delta)] \tau=B, \quad \tau \in \tilde{D}$, can be written for any $n>0$ in the form $\tau=\sum_{k=0}^{\infty} \tilde{\mathbf{R}}_{n}^{k+1} \Delta^{k} B$ where $\tilde{\mathbf{R}}_{\mu}$ is the resolvent of $\mathbf{M}$.

Acknowledgment. The authors wish to thank Prof. M. H. Bloom for his encouragement and helpful discussions during the course of this research.

\section{REFERENCES}

[1] S. G. Rubin, Incompressible flow along a corner, J. Fluid Mech. 26, 97-110 (1966)

[2] S. G. Rubin and B. Grossman, Viscous flow along a corner. II: Corner layer solution, Quart. Appl. Math. (to appear)

[3] A. Pal, A complete asymptotic expansion for the laminar flow over a flat plate, Polytechnic Institute of Brooklyn, 1970 (to appear)

[4] G. Carrier, The boundary layer in a corner, Quart. Appl. Math. 4, 367-378 (1947)

[5] J. R. A. Pearson, Homogeneous turbulence and laminar viscous flow, $\mathrm{Ph}$. D. Thesis, Cambridge University, 1957

[6] K. Stewartson, On asymptotic expansions in the theory of boundary layers, J. Math. Phys. 36, 173-191 (1957)

[7] L. Rosenhead (editor), Laminar boundary layers, Clarendon Press, Oxford, 1963

[8] P. A. Libby and H. Fox, Some perturbation solutions in laminar boundary layer theory. I: The momentum equation, J. Fluid Mech. 17, 433-439 (1963)

[9] R. Courant and D. Hilbert, Methods of mathematical physics. Vol. I, Interscience, New York 1953

[10] N. Dunford and J. T. Schwartz, Linear operators. II. Spectral theory. Self-adjoint operators in Hilbert space, Interscience, New York, 1963 